\title{
EL PAPEL DE LAS TAREAS ACADÉMICAS EN LA DINÁMICA EMOCIONAL DE ESTUDIANTES UNIVERSITARIOS. UN ESTUDIO EN CARRERAS DE EDUCACIÓN
}

\section{The role of academic tasks in emotional dynamics of undergraduates. A study in Education Careers}

\author{
PAOLA VERÓNICA PAOLONI ${ }^{1}$ \\ TATIANA LOSER ${ }^{2}$ \\ ROCÍO FALCÓN ${ }^{3}$ \\ CONICET-Universidad Nacional de Río Cuarto. Argentina. \\ Correspondencia: paopaoloni17@hotmail.com, \\ tatiloser@hotmail.com,rocio_f28@hotmail.com
}

Recibido: 20-04-2017

Revisado: 19-09-2017

Aceptado: 19-09-2017

Resumen: Este trabajo se propone brindar aportes teóricos y sugerir líneas de actuación que contribuyan al surgimiento de estados emocionales beneficiosos para los aprendizajes de estudiantes universitarios. Se trabajó con 38 alumnos de una universidad pública ubicada en la provincia de Córdoba, Argentina, durante el primer cuatrimestre de 2016. Se recurrió al Achivement Emotions Questionnaire de Pekrun, Goetz y Perry (2005), a entrevistas semiestructuradas y al análisis de protocolos de tareas académicas como instrumentos y métodos para la recolección de datos. Los resultados permiten ampliar el abanico de herramientas teóricas y metodológicas con que se cuenta para el diseño de situaciones potencialmente promotoras de emociones positivas para los aprendizajes en el contexto universitario.

Palabras clave: aprendizajes; universidad; estudiantes universitarios; tareas académicas; emociones académicas.

\begin{abstract}
This paper aims to provide theoretical contributions and suggest lines of action that contribute to the emergence of emotional states beneficial to the learning of university students. We worked with 38 students from a public university located in the province of Cordoba, Argentina, during the first four months of 2016. The instruments and methods used for data collection were the Achivement Emotions Questionnaire by Pekrun, Goetz and Perry (2005), semi-structured interviews and analysis of protocols of academic tasks. The results broaden the range of theoretical and methodological tools that are available for the design of situations potentially promoting positive emotions for learning in the university context.
\end{abstract}

Keywords: learnings; college; undergraduates; academic tasks; academic emotions.

\footnotetext{
${ }^{1}$ Investigadora en el CONICET (Argentina) y docente en la Universidad Nacional de Río Cuarto (UNRC, Argentina). Doctora en Psicología y directora del Laboratorio de Monitoreo e Inserción de Graduados de la Facultad de Ingeniería (UNRC). Líneas de trabajo: motivación académica, emociones en contextos educativos formales y rasgos de ambientes instructivos promisorios para el compromiso con metas de aprendizaje.

${ }^{2}$ Licenciada en Psicopedagogía. Becaria del Consejo Interuniversitario Nacional (Argentina). Ayudante de segunda en Intervenciones Psicopedagógicas en Instituciones Educativas de la Facultad de Ciencias Humanas de la UNRC (Argentina). Líneas de trabajo: los aspectos emocionales implicados en los aprendizajes académicos.

${ }^{3}$ Licenciada en Psicopedagogía. Integrante del Laboratorio de Monitoreo de Inserción de Graduados de la Facultad de Ingeniería de la Universidad Nacional de Río Cuarto (Argentina). Líneas de trabajo: los rasgos del contexto instructivo vinculados con la promoción de vocaciones emprendedoras en alumnos universitarios.
} 


\section{INTRODUCCIÓN}

El bajo rendimiento advertido en general en las aulas universitarias, sumado a la abulia y escasa motivación por aprender que parece caracterizar a muchos estudiantes, es un problema genuino que afecta no solo a escuelas de nivel medio de escolaridad sino también a institutos superiores universitarios y no universitarios de la República Argentina ${ }^{1}$. Así, y entre otros aspectos, la problemática referida acentúa el desafío de intervenir para mejorar la calidad de la educación de los ciudadanos. En tal sentido, conocer más acerca de los recursos internos y externos con que cuentan los estudiantes para aprender y analizar el modo en que repercuten en el compromiso asumido con el estudio es, sin duda, un modo de avanzar hacia contribuciones capaces de redundar en mejoras educativas.

Existe acuerdo en considerar que las emociones desempeñan un papel fundamental para el logro de las metas de formación en tanto son una importante variable interviniente en los aprendizajes académicos (Bar-On citado en CognitiveBehavioural, 2012; Schutz y Pekrun, 2007). Parece pertinente atender entonces a las vinculaciones que se establecen entre las emociones académicas y los rasgos de los contextos formales de aprendizaje en los que se suscitan.

Esta investigación representa una línea incipiente pero promisoria de estudio dentro del campo de la Psicología Educacional. Nadie duda de la importancia de las emociones en el devenir de los aprendizajes; no obstante, su estudio en escenarios genuinos de aprendizaje es novedoso y necesario.

Esta publicación se propone brindar aportes teóricos y sugerir líneas de actuación que contribuyan al logro de metas educativas y el surgimiento de estados emocionales beneficiosos para los aprendizajes de estudiantes universitarios. Específicamente pretende: 1) identificar las principales emociones académicas que acompañan la actuación de un grupo de alumnos universitarios en contextos genuinos de aprendizaje; 2) describir los rasgos que caracterizan a las tareas académicas propuestas en el marco de las asignaturas consideradas para este estudio; 3) conocer las percepciones y valoraciones de los estudiantes respecto de las tareas académicas que se les propone realizar; y 4) analizar las vinculaciones que se establecen entre aspectos emocionales y aspectos contextuales en la configuración de situaciones de aprendizaje.

\section{CONSIDERACIONES CONCEPTUALES}

El trabajo llevado a cabo se fundamentó en dos principales líneas de investigación: estudios sobre emociones académicas y estudios sobre contextos de aprendizaje.

\footnotetext{
1 Noticias que refieren a la falta de motivación, interés o compromiso con los estudios por parte de alumnos universitarios o del secundario son moneda corriente en Argentina. Véase por ejemplo "Preocupa la falta de motivación" (2002) y Sánchez (2013).
} 


\section{Emociones académicas}

Schutz y Pekrun (2007) denominan "emociones académicas" a las emociones que están vinculadas al contexto académico y las conciben como procesos psicológicos complejos con componentes afectivos, cognitivos, motivacionales y expresivos. Estas emociones se basan, principalmente, en el modo en que profesores y alumnos perciben lo que está ocurriendo en el contexto de una actividad concreta (Meyer y Turner citado en González Fernández, Rinaudo y Donolo, 2010).

La teoría del control-valor de las emociones de logro de Pekrun (2000, en Pekrun, Frenzel, Goetz y Perry, 2007) provee una perspectiva socio-cognitiva de las emociones de estudiantes y docentes y representa una alternativa integral a la fragmentación y dispersión teórica que caracteriza al campo de estudio de las emociones en educación en la actualidad. Paoloni (2014) considera que la teoría de Pekrun es integral en, al menos, dos sentidos complementarios: primero, porque integra una diversidad de aspectos personales relacionados con diferentes constructos estudiados en Psicología Educacional -como creencias de control de los aprendizajes, valoraciones, expectativas de resultado, creencias de autoeficacia, entre otras-; y segundo, porque integra aspectos personales y contextuales en la consideración de procesos emocionales de estudiantes y docentes.

La propuesta de Pekrun et al. (2007) sostiene que hay dos grupos de percepciones relevantes para la activación de emociones de logro en estudiantes y docentes: el control subjetivo de las actividades y de los resultados, y el valor subjetivo otorgado a esas actividades y resultados.

En cuanto al control subjetivo de actividades y de resultados, sintéticamente podemos decir que depende de las expectativas y de las atribuciones de causalidad que implican apreciaciones de control. Así, a mayor percepción de control subjetivo sobre las actividades de aprendizaje y resultados obtenidos - $\mathrm{o}$ a obtener-mayores posibilidades de experimentar emociones positivas (como disfrute, orgullo, esperanza, satisfacción, etc.). Con respecto al segundo grupo de percepciones sobre los que se fundamenta la teoría de Pekrun (citado en Pekrun et al., 2007) —esto es, el valor subjetivo otorgado a las actividades y a los resultados-, se postula una premisa similar a la del control percibido; es decir, a mayor valoración de las actividades propuestas o de los resultados obtenidos o esperados, mayores posibilidades de activar una dinámica emocional beneficiosa para los aprendizajes. Cabe aclarar que Pekrun et al. (2007) propuso un instrumento para valorar emociones discretas - tanto de valencia positiva como negativa - activadas por los estudiantes en relación con determinadas situaciones de aprendizaje (clases, tareas escolares, instancias de evaluación). Precisamente estas emociones y su dinámica es lo que consideramos en este trabajo basándonos -como veremos en el apartado metodológico correspondiente- en un cuestionario específicamente propuesto por Pekrun como fundador de la teoría del control-valor de las emociones de logro. En los trabajos de Paoloni y Rinaudo (2015) y Vaja y Paoloni 
(2012) encontramos antecedentes específicamente basados en la teoría de Pekrun y en el cuestionario que el autor propone para conocer más acerca de las emociones que estudiantes universitarios experimentan en situaciones genuinas de aprendizaje.

\section{Contextos de aprendizajes: tareas académicas como una dimensión específica}

Una revisión de antecedentes permite suponer que a lo largo de la historia de la investigación en Psicología Educacional el contexto no fue siempre entendido de la misma manera ni considerado igualmente importante respecto de los aprendizajes logrados por los estudiantes. Así, por ejemplo, en las últimas décadas se advierte un paulatino desplazamiento de visiones que tendieron a dicotomizar los vínculos existentes entre persona y situación, hacia perspectivas más integrales que atienden a la complejidad de la persona actuando en situación y al proceso de contextualización como clave para comprender el compromiso asumido para con los aprendizajes (Rinaudo, 2014).

Entre las perspectivas del contexto académico que focalizan las interacciones recíprocas que se dan entre persona y situación, se pueden mencionar fundamentalmente dos: las aproximaciones socio-cognitivas y las corrientes socioculturalistas (Perry, Turner y Meyer, 2006). Desde estas perspectivas, el contexto no es considerado como algo que permanece ajeno al sujeto. Por el contrario, desde el punto de vista de estos modelos contextualistas del aprendizaje, la metáfora del contexto como "un tejido que envuelve" o "algo que entrelaza" permite capturar en parte el carácter dinámico que se establece en el complejo sistema de la persona más la situación (Cole, 1999; Rinaudo, 2014); donde cada elemento resulta de alguna manera configurado, definido, influido, modificado en su interacción con el otro. En efecto, desde estas visiones la percepción y la acción, la emoción y el aprendizaje no son propiedades atribuibles a un individuo o a un ambiente solamente, sino más bien propiedades de un sistema que comprende a la persona actuando en situación (Barab y Plucker, 2002).

Las investigaciones acerca de la influencia del contexto en los aprendizajes han seguido principalmente estudios de Epstein (citado en Huertas, 1997; también en Alexander, 2006) y de Ames (1992), que sintetizan los hallazgos encontrados al respecto. El acrónimo TARGET propuesto por Epstein (citado en Alexander, 2006) identifica seis dimensiones de la clase que parecen tener reconocida incidencia en el compromiso asumido para con los aprendizajes; ellas son: tarea, autoridad/autonomía, reconocimiento, grupo, evaluación y tiempo. Si bien se cuenta con antecedentes de estudios que focalizaron en el reconocimiento y en la evaluación como dos de las dimensiones propuestas desde el modelo de Epstein (ver, entre otros, Paoloni y Rinaudo, 2014; Rinaudo y Paoloni, 2013), en esta oportunidad conviene detenerse particularmente en la primera de las dimensiones integradas en el acrónimo TARGET —esto es, la tarea- por la importancia que reviste respecto de los objetivos del trabajo. 
En 1989, Winne y Marx definieron a las tareas académicas como aquellos "eventos de la clase que proporcionan oportunidades para que los estudiantes usen sus recursos cognitivos y motivacionales al servicio del logro de metas personales y educacionales" (p. 242). Al respecto, Rinaudo (1999) y Rodríguez (2003) consideran que las tareas escolares no solamente refieren a esta dimensión observable del evento diseñado por el docente, sino que además constituirían la interpretación que los alumnos realizan de lo que tienen que hacer o de lo que se espera que hagan.

En los últimos años se ha extendido la noción de tarea académica. En general, era entendida como un contexto independiente capaz de motivar o emocionar a los estudiantes y comprometerlos con altos niveles de pensamiento si reunían características apropiadas. Ahora, la visión de la tarea académica como contexto de aprendizaje se ha complejizado, sus características se interpretan como dinámicas y no estáticas, en permanente interacción con factores personales y contextuales y no deterministas en sus efectos potenciales, lo que significa que los rasgos de una tarea no necesariamente redundarán en beneficios motivacionales o en la activación de emociones positivas en los estudiantes por el solo hecho de cumplir con lo teóricamente estipulado (Paoloni, 2010).

No obstante, la teoría y la investigación proveen de alguna orientación respecto de las características que deberían reunir las tareas académicas si aspiran a fomentar en los estudiantes una orientación motivacional hacia metas de aprendizaje (Paoloni, 2010). Entre las características referidas se pueden mencionar las siguientes: significatividad, instrumentalidad, moderado nivel de dificultad, curiosidad, fantasía, colaboración, posibilidad de elección y de control.

En cuanto a la significatividad, Urdan y Turner (2005) consideran que la posibilidad de otorgar sentido o significatividad a la realización de una tarea estaría vinculada a altos niveles de motivación. Son numerosos los estudios que han demostrado el valor que desempeña el interés de los estudiantes en sus respectivos procesos de aprendizaje. Una de las principales líneas de investigación sobre interés académico —el interés situacional- considera importante tener en cuenta las condiciones ambientales que pueden contribuir a que los estudiantes perciban el valor de interés de una tarea, ampliando así las posibilidades de que resulte significativa.

Por su parte, la instrumentalidad, entendida como la percepción del valor de utilidad de una tarea para el logro de metas futuras personalmente valoradas, se relacionaría también positivamente con la promoción de una orientación motivacional hacia metas de aprendizaje (González Fernández, 2005). En este sentido, a mayor percepción del valor de utilidad de una tarea respecto de las metas educativas y/o profesionales que se haya propuesto lograr un estudiante, mayores serán las posibilidades de que este alumno se implique o comprometa con el desarrollo de la actividad propuesta. 
En cuanto al nivel de dificultad de una tarea académica, la literatura especializada sobre el tema sugiere que debe ser el óptimo respecto de las capacidades de los alumnos; es decir, la tarea no debería ser tan difícil que produzca ansiedad ni tan fácil que produzca aburrimiento (Csikszentmihalyi, 1998). Parece que tareas de dificultad intermedia, entendidas como aquellas que los estudiantes pueden llevar a cabo únicamente con algo de esfuerzo, se presentan como la mejor alternativa para comprometerlos con sus aprendizajes.

Atendiendo a la curiosidad y a la fantasía como otros rasgos del contexto potencialmente promotores de una orientación motivacional hacia metas de aprendizaje, la teoría sugiere que las tareas o actividades que resultan novedosas e imprevisibles estimularían en los estudiantes curiosidad, interés y conductas de exploración, fomentando de este modo un genuino compromiso con los estudios. Por su parte, las actividades que impliquen a los estudiantes en la ilusión y en el hecho de hacer algo a través de simulaciones o juegos, pueden ser promotoras también de una orientación motivacional dirigida a metas de aprendizaje (Pintrich y Schunk, 1996).

En relación con la posibilidad de colaboración que ofrece una tarea académica, Huertas y Montero (2001) sostienen que aquellos escenarios educativos que permiten a los alumnos trabajar en grupos igualitarios y de confianza mutua son propicios para generar una adecuada aceptación social y una orientación motivacional dirigida a metas de aprendizaje.

Finalmente, entre las características de las tareas académicas mencionadas como potencialmente promotoras de una orientación motivacional hacia metas de aprendizaje, la elección y la posibilidad de control de la actividad se presentan como las que logran mayores acuerdos por parte de los investigadores contemporáneos sobre motivación en educación. Así pues, autores como Ames (1992), Pintrich y Schunk (1996), Ryan y Decy (2000) - entre muchos otros - , coinciden en sugerir que en la medida que una persona experimenta mayor sentimiento de autodeterminación o control en la realización de alguna tarea, mayor será su motivación intrínseca, su interés, los esfuerzos desplegados por obtener resultados óptimos, el placer asociado a la realización de la actividad emprendida y las posibilidades de obtener mejores logros académicos. En tal sentido, las tareas que cumplan con esta condición favorecerían en los estudiantes el sentimiento de autodeterminación y la creencia de estar "a cargo" o tener cierto control sobre los resultados.

\section{CONSIDERACIONES METODOLÓGICAS}

Este estudio está orientado por los lineamientos de la investigación de diseño. El diseño instructivo se elabora, implementa y somete a escrutinio de investigación (Rinaudo y Donolo, 2010). Se hace uso de los resultados de la investigación aplicada para mejorar las intervenciones, posibilitando la deliberación y negociación de los problemas de la práctica y de la participación. 
Según Stake (citado en Barone, 2004) este estudio de diseño específicamente se focaliza en un estudio de caso instrumental en tanto lo que se busca es lograr insight en relación con el tema que se convoca.

\section{Participantes}

Durante el ciclo lectivo 2016 se trabajó con todos los alumnos que cursaron Didáctica $(\mathrm{N}=38)$, asignatura cuatrimestral presente en los planes de estudios del Profesorado y Licenciatura en Educación Especial y de la Licenciatura en Psicopedagogía de una universidad pública de la provincia de Córdoba, República Argentina. Los alumnos que cursaron esta asignatura son avanzados en sus respectivos planes de formación en tanto esta materia se propone en el penúltimo año de la cursada.

\section{Instrumentos y modalidades de recolección de datos}

Los datos necesarios fueron recabados por medio de diversos instrumentos y modalidades de recolección, a saber: Achivement Emotions Questionnaire (AEQe) de Pekrun, Goetz y Perry (2005, en la versión traducida al español y validada por Paoloni, Vaja y Muñoz, 2014); entrevistas semiestructuradas; y análisis de protocolos de tareas académicas solicitadas como parte del proceso de formación de los estudiantes.

El instrumento AEQe consta de diversas secciones. A los fines de este trabajo se consideró únicamente la sección que evalúa emociones vinculadas con la clase, teniendo en cuenta tres momentos: antes, durante y después de asistir a las clases de las asignaturas en las que se administró el instrumento. La sección que utilizamos en esta oportunidad está compuesta por 80 ítems que se responden considerando una escala Likert de 5 puntos en la que los estudiantes indican su grado de acuerdo o desacuerdo de esas afirmaciones. De esta manera la puntuación más baja (1) indica un bajo grado de acuerdo con la afirmación y la puntuación más alta (5) indica un total acuerdo con la afirmación. Los ítems de esta sección se agrupan en 8 escalas: disfrute, esperanza, orgullo, enfado, ansiedad, vergüenza, desesperanza y aburrimiento.

Disfrute: compuesto por 10 ítems que aluden al disfrute como un sentimiento agradable de gozo o complacencia en respuesta a un estímulo. Un estudiante que disfruta de las clases a las que asiste sería aquel que se entusiasma por ir y se siente complacido por participar de esas clases.

Esperanza: compuesta por 8 ítems que refieren a una emoción agradable que se experimenta cuando percibimos como posible aquello que deseamos. Un estudiante siente esperanza en relación con las clases cuando considera que puede desempeñarse correctamente en la asignatura y lograr las metas que se proponga.

Orgullo: compuesta por 9 ítems que aluden a un alto nivel de estimación propia. Un estudiante que experimenta orgullo sería aquel que se siente conforme respecto de sus desempeños y logros obtenidos. 
Enfado: compuesto por 9 ítems que aluden a una emoción desagradable contra alguien $\mathrm{o}$ algo. Un estudiante que experimenta enojo en relación con las clases a las que asiste sería aquel que se irrita o enfada ante ciertas características de esas clases.

Ansiedad: compuesta por 12 ítems que hacen referencia a un estado de agitación, preocupación o inquietud. Un estudiante que experimenta ansiedad antes, durante o después de asistir a clases estaría preocupado o alterado por diferentes pensamientos capaces de distraerlo.

Vergüenza: compuesta por 11 ítems que hacen referencia a una emoción desagradable, de retraimiento o inhibición que se experimenta ante determinadas situaciones. Un estudiante que siente vergüenza sería aquel que se inhibe en las clases por timidez o por percibir que cometió una acción indebida.

Desesperanza: compuesta por 10 ítems. En este sentido, un estudiante desesperanzado sería aquel que no siente esperanza de alcanzar u obtener los logros propuestos o deseados en el marco de las clases.

Aburrimiento: compuesta por once ítems que refieren al sentimiento desagradable de cansancio o tedio que se experimenta generalmente ante situaciones percibidas como un sinsentido y que dificulta prestar atención o involucrarse en las tareas requeridas. Así, se supone que un alumno aburrido en clase tendrá problemas para prestar atención, concentrarse, percibir el sentido de lo requerido, esforzarse y persistir ante las dificultades.

Siguiendo las indicaciones de Pekrun et al. (citado en Mouratidis, Vansteenkiste, Lens y Vanden-Auweele, 2009), para este estudio se agruparon las escalas del AEQe según valencia y activación en tres subescalas: la primera corresponde a emociones de activación positiva e incluye disfrute, esperanza y orgullo (27 ítems en total). La segunda integra emociones de activación negativa e incluye enojo, ansiedad y vergüenza (32 ítems en total). Finalmente, la tercera refiere a emociones de desactivación negativa e incluye desesperanza y aburrimiento (21 ítems en total).

Entrevistas semiestructuradas. En el marco de las entrevistas, primero, se agradeció a los estudiantes por su participación, se realizó una presentación de los entrevistadores y de los objetivos de la entrevista, así como una contextualización en el marco del proyecto de investigación. También se advirtió acerca de la confidencialidad de los datos compartidos. En segundo lugar, se indagó acerca de las emociones académicas que los alumnos recordaban haber experimentado en el contexto de las clases de Didáctica, así como sus percepciones acerca de los rasgos del contexto instructivo que para ellos se asociaban al surgimiento de emociones beneficiosas para los aprendizajes.

Las entrevistas se realizaron de manera individual, a un total de 18 alumnos seleccionados al azar y atendiendo a su predisposición y disponibilidad para participar en la investigación. La duración promedio de cada entrevista fue entre 20 y 30 minutos. El período en que se llevaron adelante fue entre junio y agosto del 2016. 
Análisis de documentos. Se consideró el análisis de las consignas de dos tareas que fueron diseñadas particularmente con la intención de promover en los estudiantes una motivación orientada a metas de aprendizaje y una dinámica emocional óptima. El análisis de los protocolos o consignas de trabajo se efectuó desde las dimensiones contextuales revisadas y extendidas por Paoloni (2010).

\section{Procedimiento}

A comienzo de las clases del ciclo lectivo 2016 se informó a los alumnos de Didáctica sobre el interés de la cátedra en implementar una investigación que analizara la dinámica de aspectos emocionales considerados en situaciones genuinas de aprendizaje. Se invitó a los alumnos a participar voluntariamente al tiempo que se les aclaró sobre la confidencialidad de los datos proporcionados en el marco del estudio referido. Todos los estudiantes decidieron participar. Transcurrida la segunda mitad del cuatrimestre (octubre 2016) los alumnos respondieron al AEQe. Luego, se analizó desde criterios teóricos preestablecidos las consignas de las tareas académicas solicitadas a los alumnos como parte de su proceso de formación. Las tareas requeridas formaron parte del diseño instructivo que en este estudio se sometió a valoración (en la que consideramos los rasgos que, en teoría, se vinculan con la promoción de compromiso académico por parte de los estudiantes) y que tuvo como objetivo promover en los estudiantes el desarrollo de metas de aprendizaje y una dinámica emocional óptima a tal fin. En tercer lugar - momento de finalización de las clases- se seleccionó al azar un subgrupo de 18 alumnos para ser entrevistados (esto es, casi el $50 \%$ del total de participantes).

Los datos fueron analizados desde procedimientos cualitativos y cuantitativos. Se empleó el programa SPSS (versión 18.0) para el procesamiento y análisis de datos recabados mediante el AEQe. Los datos que se recabaron mediante análisis de documentos y entrevistas semiestructuradas fueron analizados según procedimientos idóneos vinculados a metodologías cualitativas. Específicamente para el caso de las entrevistas se consideraron las orientaciones proporcionadas desde la teoría fundada 0 Grounded Theory (Glaser y Strauss, 1967) que permitió conformar categorías emergentes a partir de los datos. Las consignas de las tareas por su parte se analizaron en base a criterios teóricos definidos a priori y sistematizados en Paoloni (2010).

\section{RESULTADOS}

Los resultados obtenidos se organizan en tres apartados: 1) emociones vivenciadas por los estudiantes en relación con las clases; 2 ) rasgos contextuales que caracterizaron a las tareas académicas solicitadas en el marco de las asignaturas; y 3) percepciones y valoraciones de los alumnos sobre las tareas requeridas. 


\section{Emociones vivenciadas por los estudiantes en relación con las clases}

La Tabla 1 sistematiza los resultados obtenidos por los alumnos en cada una de las escalas de la sección del AEQe que evalúa emociones relacionadas con las clases.

\begin{tabular}{ccccc} 
Tabla 1: Puntuaciones media, mediana, moda $y$ desviación estándar para cada escala \\
$\begin{array}{c}\text { sección clases. Datos para } 38 \text { alumnos de Didáctica. Año } 2016 . \\
\text { Universidad pública de la Provincia de Córdoba, Argentina. }\end{array}$ \\
\hline Emociones & $\mathbf{M}$ & Mdn & Mo & D.S \\
\hline Disfrute & 3,14 & 3,10 & 2,70 & 0,51 \\
Esperanza & 3,26 & 3,25 & 3,25 & 0,65 \\
Orgullo & 2,86 & 3,00 & 3,00 & 0,65 \\
Enfado & 1,70 & 1,67 & 1,67 & 0,55 \\
Ansiedad & 2,07 & 1,92 & 1,42 & 0,74 \\
Vergüenza & 1,88 & 1,64 & 1,45 & 0,70 \\
Desesperanza & 1,61 & 1,55 & 1,60 & 0,53 \\
Aburrimiento & 2,37 & 2,23 & 1,82 & 0,69 \\
& & & & \\
\hline
\end{tabular}

Fuente: Elaboración propia (2017)

Si se considera que la escala de puntuación del AEQe varía entre 1 y 5 , entonces los datos indican que los alumnos de este grupo obtuvieron puntajes intermedios en emociones como disfrute $(M=3,14)$, esperanza $(M=3,26)$ y orgullo $(M=2,86)$. Por su parte, emociones como enfado $(M=1,70)$, ansiedad $(M=2,07)$, vergüenza $(M=1,88)$, desesperanza $(M=1,61)$ y aburrimiento $(M=2,37)$ obtuvieron un promedio bajo.

Si se agrupan las escalas según grado de activación y valencia, los resultados muestran detalles importantes. Como se observa en la Tabla 2, los alumnos obtuvieron puntajes intermedios en escalas que consideran emociones de activación positiva (disfrute, esperanza y orgullo). Para las escalas que evalúan emociones de activación negativa (enfado, ansiedad y vergüenza) y de desactivación negativa (desesperanza y aburrimiento) los puntajes promedio fueron bajos. Por lo tanto, esta clasificación muestra que los estudiantes manifestaron mayormente emociones positivas en relación con las clases durante el cursado, lo que teóricamente favoreció su predisposición para comprometerse con las materias y la promoción de desempeños académicos óptimos. 
Tabla 2: Puntuaciones medias para las escalas de emociones de activación positiva, activación negativa y desactivación negativa. Datos para 38 alumnos de Didáctica. Año 2016. Universidad pública de la Provincia de Córdoba, Argentina.

\begin{tabular}{cc}
\hline Emociones & Puntuación media \\
\hline Emociones de activación positiva & 3,09 \\
Emociones de activación negativa & 1,88 \\
Emociones de desactivación negativa & 1,99 \\
\hline
\end{tabular}

Fuente: Elaboración propia (2017)

Para profundizar en el análisis de datos se agruparon los puntajes de las diferentes escalas según el momento en relación con el cursado de las asignaturas intervinientes en este estudio; así, se consideraron las frecuencias de mención para cada emoción antes, durante y después de asistir a clases. Los resultados se exponen en la Tabla 3.

\begin{tabular}{|c|c|c|c|c|c|c|}
\hline \multirow{2}{*}{ Emociones } & \multicolumn{2}{|c|}{ Antes de las clases } & \multicolumn{2}{|c|}{ Durante las clases } & \multicolumn{2}{|c|}{ Después de las clases } \\
\hline & M & D.S. & M & D.S. & M & D.S. \\
\hline Disfrute & 1,69 & 0,38 & 2,99 & 0,68 & 3,09 & 0,58 \\
\hline Esperanza & 3,23 & 0,65 & 3,47 & 0,95 & - & - \\
\hline Orgullo & - & - & 2,67 & 0,73 & 3,10 & 0,72 \\
\hline Enfado & 1,81 & 1,06 & 1,58 & 0,63 & 1,80 & 0,62 \\
\hline Ansiedad & 2,11 & 0,75 & 2,02 & 0,89 & - & - \\
\hline Vergüenza & - & - & 1,93 & 0,75 & 1,66 & 0,82 \\
\hline Desesperanza & 1,56 & 0,50 & 1,74 & 0,71 & 1,54 & 0,82 \\
\hline Aburrimiento & - & - & 2,37 & 0,68 & - & - \\
\hline
\end{tabular}

Fuente: Elaboración propia (2017) 
De acuerdo con los datos presentados en la Tabla 3, las emociones experimentadas antes de asistir a las clases de las asignaturas en la que se llevó a cabo este estudio, la esperanza fue la emoción experimentada con mayor intensidad $(M=3,23)$. La desesperanza, por el contrario, sería la emoción menos experimentada por los alumnos en momentos previos a asistir a clases $(M=1,56)$.

Por su parte, durante la asistencia a clases de las asignaturas consideradas para este estudio, los datos muestran que la emoción experimentada con más intensidad por los alumnos sigue siendo la esperanza en primer lugar $(M=3,47)$, seguida de cerca por el disfrute $(M=2,99)$ y el orgullo $(M=2,67)$. Con menor intensidad en cambio, los alumnos experimentaron aburrimiento $(M=2,37)$, ansiedad $(M=2,02)$, vergüenza $(M=1,93)$, desesperanza $(M=1,74)$ y enfado $(M=1,58)$.

Finalmente, después de asistir a clases, las puntuaciones obtenidas sugieren que las emociones vivenciadas con mayor intensidad por los alumnos fueron el orgullo $(M=3,10)$ y el disfrute $(M=3,09)$, no habiendo prácticamente diferencia entre ambas. Las tres emociones experimentadas en menor intensidad por los alumnos después de las clases fueron el enfado, la vergüenza y la desesperanza.

En definitiva, si se atiende a la dinámica de las emociones experimentadas por los alumnos en relación con la asistencia a clases, se advierte que emociones como enfado y desesperanza tendieron a mantenerse en niveles bajos, en comparación con el disfrute, la esperanza y el orgullo que mostraron puntajes promedio altos. Además, es interesante destacar cómo las emociones de activación positiva obtuvieron puntajes que fueron en ascenso durante los tres momentos considerados en este estudio. Por ejemplo, para disfrute se obtuvo $M=1,69$ antes de las clases, $M=2,99$ durante las clases y $M=3,09$ después de las clases. Mientras que emociones como la desesperanza y la vergüenza muestran un paulatino descenso si se comparan sus puntuaciones promedio durante y después de la asistencia a clases.

\section{Acerca de los rasgos que caracterizan a las tareas académicas propuestas en el marco de las asignaturas consideradas en este estudio}

En este apartado se analizan las tareas académicas propuestas en el marco de las asignaturas intervinientes en este estudio. Se solicitaron dos tareas principales. La primera consistió en realizar una entrevista a un profesional graduado de la misma carrera que los alumnos cursaban. La segunda tarea consistió, a grandes rasgos, en la realización de una secuencia didáctica pensada para ser implementada en un contexto no formal de aprendizaje. Ambas tareas contaban con lineamientos generales sobre su realización y formaban parte de la propuesta de formación profesional de los estudiantes: para el caso de la entrevista, se trató de un trabajo práctico obligatorio y para el caso de la secuencia didáctica, se trató de un examen parcial para aspirar a lograr la promoción o la regularidad de la materia. 
Un análisis de las consignas de las tareas requeridas a los alumnos permite identificar algunos rasgos que, en teoría, se vinculan con la promoción de compromiso académico por parte de los estudiantes, así como con el despliegue de una dinámica emocional beneficiosa para sus aprendizajes. A continuación, se analiza con mayor detenimiento cada uno de los rasgos contextuales que en teoría caracterizaron a estas tareas, basándose principalmente en la sistematización de investigaciones que al respecto realizó Paoloni (2010).

Variedad y diversidad: las tareas requeridas a los participantes de este estudio fueron multidimensionales en el sentido que priorizaban variedad de procedimientos con iguales posibilidades de resultados correctos. Los alumnos tenían autonomía y libertad para decidir varios aspectos durante el proceso de elaboración de la tarea, lo que obviamente impactó en la diversidad y variedad de los productos resultantes. Así por ejemplo, para el caso de la entrevista a un profesional, los estudiantes tenían libertad para elegir los compañeros con los que trabajar, el profesional a quien entrevistar, las preguntas a realizar, el contexto no formal para el desempeño del rol que quisieran considerar - tribunal, cárcel, geriátrico, universidad, consultorio, entre otros-, el modo de organizarse para llevar a cabo el trabajo - horarios, espacios-. Además, podían elegir la modalidad que más se ajustara a sus intereses y posibilidades para compartir lo realizado con sus compañeros en el contexto del aula. Algo similar sucedió con la segunda actividad requerida — la secuencia didáctica—, que brindaba un amplio margen de autonomía a los alumnos para definir aspectos estructurantes.

Significatividad: se entiende que ambas tareas eran potencialmente significativas en tanto brindaban amplias posibilidades a los estudiantes para establecer relaciones con sus intereses, conocimientos previos, experiencias, metas, proyectos profesionales, etc. Así, en teoría, las tareas requeridas promovían la construcción de aprendizajes con sentido.

Instrumentalidad: si la instrumentalidad o el valor de utilidad de una tarea está dado por las "vinculaciones" que la persona puede establecer entre el objetivo de la tarea y sus metas futuras de desempeño profesional (Alonso Tapia, 2000), entonces se entiende que en teoría las tareas solicitadas a los estudiantes como parte de sus procesos de formación fueron potencialmente instrumentales, en tanto promovían una ampliación del imaginario ocupacional y la diversificación de las representaciones acerca del rol profesional.

Fantasía: ambas tareas solicitadas permitían en teoría el despliegue de fantasía por parte de los alumnos. En el caso de la entrevista al profesional, por ejemplo, los estudiantes podían imaginarse a sí mismos cumpliendo (o no) el rol del entrevistado; en el caso de la secuencia didáctica los alumnos debían planificar una secuencia "como si" fueran a llevarla a cabo, apuntando a la simulación de un rol que en el terreno de la fantasía los posicionaba como profesionales. 
Colaboración: ambas tareas eran grupales por lo que promovían en teoría un trabajo cooperativo. Así, se entiende que el espacio de la tarea brindaba posibilidad para intercambiar diferentes opiniones, expresar y conocer diferentes puntos de vista, defender posturas, fundamentar posiciones y compartir el fruto del trabajo en equipo con el resto de la clase. Se trata sin duda de un aspecto del contexto que resultados de investigación destacan especialmente como importante por su notable influencia en la dinámica emocional de los estudiantes, así como en su motivación para aprender (Huertas y Montero, 2001).

Elección y control: tanto en la tarea de entrevistar a un profesional como en la de diseñar una secuencia didáctica los alumnos contaban con un amplio margen de autonomía que reconoce en teoría diferentes tipos: autonomía organizativa, autonomía procedimental, autonomía cognitiva (Stefanou citado en González Fernández, 2005).

Por lo tanto y a partir del análisis teórico que se efectuó acerca de las consignas de las tareas académicas propuestas desde Didáctica, se entiende que las mismas reunían características vinculadas con la promoción de una orientación motivacional hacia metas de aprendizaje y estados emocionales beneficiosos para tal fin.

\section{Acerca de las percepciones y valoraciones de los alumnos sobre las tareas solicitadas}

En este apartado se analizan las percepciones y valoraciones realizadas por los alumnos acerca de las tareas propuestas como parte de sus procesos de formación en el marco de las asignaturas consideradas en este estudio. Los aportes proporcionados por los alumnos en las entrevistas pueden organizarse en cuatro apartados principales: valor de utilidad de las tareas, valor de importancia de las tareas, percepción y valoración de la autonomía como rasgo positivo, y otras apreciaciones relativas a rasgos contextuales de las tareas requeridas.

Valor de utilidad de las tareas. En el caso de la entrevista a un profesional, 13 de 18 alumnos identificaron valoraciones positivas en relación con la realización de esta actividad lo cual, según sus apreciaciones, generó en ellos emociones beneficiosas para sus aprendizajes. Específicamente 10 estudiantes destacaron como aporte principal de la tarea el acercamiento que les permitió al futuro rol profesional y la posibilidad de establecer vinculaciones entre lo que se aprende en la universidad - como contenidos teóricos básicos- y lo que es necesario saber o conocer para desempeñar el rol de psicopedagogo o profesor en educación especial en contextos laborales. El valor de utilidad otorgado por los alumnos a esta tarea en particular y los estados emociones beneficiosos que parece haber activado se orientan a corroborar postulados básicos de la teoría de Pekrun (citado en Pekrun et al., 2007), según la cual las valoraciones son uno de los principales antecedentes en el surgimiento de las emociones académicas. A continuación, algunos ejemplos ilustran el sentido de lo señalado: 
[La realización de la tarea] te permite conocer un poco más del rol profesional ya que va contando su experiencia, cómo ingresó al trabajo, qué le pasó y qué no, si fue fácil o difícil y eso te va preparando y concientizando de otra forma también. Además, creo que es importante porque ya estamos casi en el tramo final de la carrera y está bueno conocer un poco más (Entrevista N4, alumna de Educación Especial).

La actividad me encantó porque también nos permite ver nuestra tarea. El campo del psicopedagogo es muy amplio y muchas veces te preguntas ¡ay, no! ¿Qué voy a hacer después de que me reciba o...? Pero hay en realidad, lo que pasa es que hay que ser creativo, pero siempre hay algo. Para mí es muy amplio el campo (...) haber entrevistado es como que vos ya ves la otra mirada de alguien que ya está ejerciendo y que ya se recibió y ya pasó por todo esto que nosotros estamos pasando (Entrevista N¹, alumna de Licenciatura en Psicopedagogía).

La actividad que propusieron como 2do parcial [secuencia didáctica] estuvo muy buena. A mí me re gustó. La verdad muy práctica. A mí personalmente me gustó mucho. Fue mucho más relacionado a la profesión de uno (Entrevista N¹7, alumna de Licenciatura en Psicopedagogía).

Valor de importancia de las tareas. Para el caso de la elaboración de la secuencia didáctica se advirtió algo similar en el análisis de los aportes brindados por los estudiantes en el marco de las entrevistas de las que participaron. Nuevamente 13 de 18 alumnos identificaron rasgos positivos de esta tarea en especial ligados al surgimiento de emociones beneficiosas para sus aprendizajes. Dentro de los aspectos positivos, los alumnos identificaron claramente el valor de importancia de esta tarea en el sentido que promovió la construcción de nuevos conocimientos, les ayudó a entender temas trabajados en clases y a establecer relaciones no arbitrarias y sustantivas entre conceptos troncales desarrollados en el contexto de la asignatura. Nuevamente las valoraciones, esta vez de significatividad, se orientan a corroborar los postulados de la teoría del control-valor de las emociones propuesta por Pekrun (citado en Pekrun et al., 2007), según la cual, a mayor valoración del contexto de aprendizaje, mayores posibilidades de activar emociones beneficiosas consecuentes. A continuación, un par de ejemplos permiten ilustrar el sentido de lo referido:

Me parece que, en el hecho de hacernos reflexionar, pensar cómo hacer, cómo llevar a la práctica el conocimiento, todo lo que venimos viendo de la teoría. Eso como que sirve un montón, para nuestra futura práctica profesional. Es como que hasta ahora yo no había tenido una actividad así en donde fuese tan específica y práctica por pensar en cómo hacerlo (Entrevista №17, alumna de Licenciatura en Psicopedagogía).

La actividad que más me gusto fue la de hacer la secuencia. No era obligatorio, pero teníamos que hacerla en un contexto no formal, en una institución no formal. Yo la hice en [nombre de la institución] me interesó y me gustó mucho, no conocía la institución ni el lugar. No era necesario, 
no te lo pedía la consigna, pero como a mí me interesó fui y hablé con la gente, con la señora que está a cargo, le hice una entrevista más o menos acerca del contexto y de la gente que va al lugar para después pensar qué secuencia didáctica podía hacer (Entrevista $N^{\circ} 9$, alumno de la Licenciatura en Psicopedagogía).

Percepción y valoración de la autonomía como rasgo positivo del contexto. Aunque este rasgo del contexto instructivo es uno de los que con mayor fuerza acentúan diversas teorías motivacionales - como, por ejemplo, la teoría de la autodeterminación de Ryan y Decy (2000) - como así también la teoría del control-valor de las emociones de Pekrun (citado en Pekrun et al., 2007), llama la atención que en este estudio solo 2 de los 18 alumnos entrevistados lo identificaran y valoraran como un rasgo contextual positivo respecto de sus aprendizajes. En palabras de los estudiantes:

Nos daban la libertad de elegir a quién se lo queríamos hacer y además después lo podías contar y te sentís identificado un poco. Nos dieron lugar en la Didáctica para poder expresar y que nos escucháramos entre todos, creo que en ese sentido (Entrevista №16, alumna de Educación Especial).

Esa [tarea] estuvo buena porque era hablar directamente con un profesional que nos brindó conocimientos de la práctica, no teórico, y después poder compartirlo en clase. La profesora nos dio ese espacio. No era que nos daba una cantidad de minutos. Fue como bastante libre, pudimos explayarnos bien y mostrar lo que habíamos averiguado nosotras (Entrevista №13, alumna de Educación Especial).

Otras apreciaciones relativas a rasgos contextuales de las tareas requeridas. Respecto de la segunda tarea los alumnos entrevistados mencionaron en diferentes frecuencias de mención algunos rasgos contextuales que valoraron de modo positivo y vincularon con estados emocionales beneficiosos para sus aprendizajes. Entre estos rasgos se destacan los siguientes: la tarea tenía lineamientos generales y flexibles que organizaban la actividad; era novedosa en el sentido de que nunca habían realizado una tarea de este tipo; formaba parte del abanico de actividades de preferencia personal; contaban con varias instancias de consulta a docentes antes de la entrega final; podía realizarse en grupo. A continuación, un ejemplo pretende ilustrar el sentido de lo referido:

Esa actividad me gustó, tiene que ver con las actividades que yo prefiero hacer (Entrevista №2, alumna de Licenciatura en Psicopedagogía).

Las ideas se me ocurrían a mí la mayoría y me gustaban, me interesaban, por eso creo que me gustó mucho esa actividad, porque fue un grupo, me motivo, me gusto leer, aprendí (...) a hacer una secuencia, (...) y me sentí guiado por la profe, por más que hayan sido pocas consultas o por mail. Había un seguimiento de las profes. (Entrevista $\mathrm{N}^{\circ} 9$, alumno de Licenciatura en Psicopedagogía). 


\section{CONSIDERACIONES FINALES: APORTES DEL ESTUDIO Y POSIBLES LIMITACIONES}

Con esta publicación nos propusimos brindar aportes teóricos y sugerir líneas de actuación que contribuyan al logro de metas educativas y el surgimiento de estados emocionales beneficiosos para los aprendizajes de estudiantes universitarios.

De acuerdo con los hallazgos obtenidos, los alumnos que participaron de este estudio manifestaron mayormente emociones positivas en relación con el cursado de la materia Didáctica, tanto en momentos previos como durante y después de la asistencia a clases. Cuando buscamos identificar los rasgos del contexto que pueden haber promovido esta dinámica emocional beneficiosa para los aprendizajes, encontramos que las principales tareas requeridas a los alumnos como parte de su proceso de formación efectivamente reunieron rasgos o pistas contextuales teóricamente promotoras de creencias motivacionales y emociones beneficiosas para los aprendizajes. Así, las tareas solicitadas se caracterizaron en general por ser instrumentales (promover valor de utilidad en relación con las metas de formación profesional), significativas (promover sentido y valor de importancia de la actividad requerida), por brindar posibilidad para trabajar en grupo, permitir despliegue de fantasía y contar con diversos espacios para una actuación autónoma que promueva el control de los procesos de aprendizajes por parte de los estudiantes. Pero cuando indagamos las percepciones y valoraciones de los alumnos respecto de estas pistas o rasgos del contexto de aprendizaje, los hallazgos se tornaron más interesantes tanto por corroborar los principales postulados de la teoría de base -teoría del control-valor de Pekrun (2000, en Pekrun et al., 2007) - como por distanciarse, en parte, de ello.

Los hallazgos que se orientan a corroborar la teoría del control-valor de las emociones son aquellos que precisamente destacan el rol protagónico de las valoraciones como mediadoras -o antecedentes - en el surgimiento de las emociones de los estudiantes. Específicamente, los alumnos de nuestro estudio lograron percibir la instrumentalidad de las tareas propuestas en relación con sus futuros desempeños profesionales (valor de utilidad), también percibieron su significatividad en tanto para ellos tuvo sentido la realización de estas tareas en el marco de los aprendizajes promovidos (valor de importancia), y percibieron asimismo como novedosas a las actividades propuestas, lo que sin duda impactó en el valor de interés suscitado hacia las mismas. En definitiva y como se advierte, los datos se orientan a corroborar la importancia que asumen rasgos contextuales como la instrumentalidad, la significatividad o la novedad por su potencial para promover entre los estudiantes valoraciones positivas respecto del contexto de aprendizaje. Estos rasgos del contexto resultaron potencialmente promotores de valoraciones de utilidad, de importancia y de interés entre los alumnos lo que, como lo muestran los hallazgos, se vinculó al 
surgimiento de una dinámica emocional beneficiosa para los aprendizajes —alto nivel de emociones positivas de activación en comparación con bajas puntuaciones para emociones negativas de desactivación o de activación-. En síntesis, los hallazgos sugieren que la dinámica emocional positiva que parece haber acompañado la actuación académica de este grupo de estudiantes puede entenderse, en parte, por el surgimiento de valoraciones positivas respecto del contexto de aprendizaje en el que particularmente se llevó a cabo la experiencia, tal y como la teoría lo sugiere. Ahora bien, ¿sucedió lo mismo con las percepciones de autonomía y de control?, ¿fueron los espacios de autonomía relativos a la elaboración de la tarea, valorados en el sentido en que la teoría lo supone? Aparentemente no.

Los resultados que no se orientan en el sentido en que lo supone la teoría, tienen que ver con una aparente dificultad que los estudiantes de este estudio tuvieron para percibir o bien para valorar positivamente la autonomía como rasgo del contexto teóricamente diseñado para promover en ellos una motivación más comprometida con metas de aprendizaje. Comentarios como los siguientes, ilustran lo referido: "siento que nos dejaron solos en la realización de la tarea"; "faltó explicar cómo se hacía, qué había que tener en cuenta"; "tuve que buscar en Internet qué era una secuencia y encima fijarme que sea una página segura"; entre otros.

En relación con este hallazgo en particular queremos efectuar dos consideraciones. Primero, este tipo de resultado no constituye un antecedente aislado, sino que se vincula con otros obtenidos en estudios previos que focalizaban en la motivación de los alumnos por aprender. En un sentido similar al advertido en este trabajo, en estudios llevados a cabo por Paoloni (2009), alumnos universitarios avanzados tuvieron también dificultades para percibir y valorar positivamente el espacio de autonomía con que contaban para realizar diferentes tareas académicas requeridas como parte de sus procesos de formación. Respecto de esta aparente dificultad de los alumnos para percibir los espacios de autonomía como un rasgo positivo del contexto de aprendizaje, nos atrevemos a pensar que elementos culturales juegan aquí un papel de relevancia que amerita el interés de futuras investigaciones. Tanto la teoría del controlvalor de las emociones de Pekrun (2000, en Pekrun et al., 2007) como la teoría de la autodeterminación de Ryan y Decy (2000), fueron creadas en contextos socioculturales diferentes a nuestra realidad argentina. En nuestra tradición educativa, los espacios para el despliegue de autonomía durante los trayectos académicos suelen ser muy reducidos. Las posibilidades con que en general cuentan nuestros estudiantes para optar entre un abanico de alternativas son mínimas. Están poco acostumbrados a elegir o ser protagonistas en la definición de los productos académicos -escritos, orales, alternativos - que se les solicita y quizás esto contribuya a explicar, en parte, los resultados obtenidos en este trabajo.

En cuanto a la segunda consideración, si los estudiantes de nuestro estudio parecen no haber percibido o bien no haber valorado los espacios de autonomía en un sentido positivo, pero a diferencia de lo que predice la teoría sus actuaciones se vieron 
impregnadas de emociones beneficiosas para los aprendizajes como el disfrute, la esperanza o el orgullo, entonces resultaría de interés matizar estas presunciones, considerando además las metas que orientan los aprendizajes de los estudiantes. En el marco de estos resultados y de hallazgos obtenidos en estudio previos (Paoloni y Rinaudo, 2015), las metas que orientan la actuación de los estudiantes podrían funcionar como potentes mediadoras motivacionales en el surgimiento de las emociones académicas. Entonces, si un alumno orienta su actuación predominantemente por metas extrínsecas o de desempeño, una tarea académica que podría entusiasmarlo sería aquella que precisamente limite la autonomía en su actuación —por ejemplo, mediante preguntas de múltiple opción-; por el contrario, una tarea que brinde autonomía y libertad para demostrar que "domina" los contenidos y que ha construido aprendizajes significativos, probablemente lo pondría tenso, nervioso, ansioso o angustiado.

En síntesis, en cuanto a las implicaciones teóricas de este estudio, entendemos que sus resultados se orientan a corroborar postulados básicos de Pekrun et al. (2007) que específicamente acentúan la importancia que asume el valor de la tarea como antecedente en el surgimiento de emociones académicas en los estudiantes. Así, los hallazgos obtenidos sugieren que el valor de importancia, de utilidad y de interés percibido por los estudiantes respecto de una tarea propuesta fue uno de los principales antecedentes en el surgimiento de emociones positivas de activación -como la esperanza, el orgullo y el disfrute- y una dinámica emocional beneficiosa a tal fin. No obstante, los hallazgos señalan que el control percibido no fue - al menos en el sentido en que la teoría lo presupone- un antecedente relevante respecto de las emociones vivenciadas por los alumnos de nuestro estudio. En este orden de ideas, resulta promisorio profundizar el alcance de esta teoría en el marco de la diversidad de contextos socioculturales en los cuales se aplica. Si hiciéramos esto, estaríamos atendiendo a una de las futuras direcciones en investigación educativa propuesta por autores como Linnenbrik-Garcia y Pattal (2016), que sugieren estudiar las diferencias transculturales en el desarrollo de teorías educacionales; específicamente proponen que investigaciones sobre el rol de la cultura en procesos psicológicos implicados en los aprendizajes -como motivación, emoción, voluntad, etc.- son particularmente necesarias e interesantes en tanto consideran de un mejor modo los múltiples componentes del contexto que sinergizan los procesos de aprendizajes y resultados obtenidos. Por otra parte, en investigaciones futuras entendemos que resulta necesario indagar más detenidamente la participación que asume la orientación a la meta en la dinámica de emociones académicas concomitantes.

En cuanto a implicaciones prácticas de este trabajo, en el marco de los resultados obtenidos podrían entenderse ciertas intervenciones orientadas a promover en los estudiantes una valoración positiva de sus espacios de autonomía y control para aprender. En sentido similar, por ejemplo, se ha propuesto el reentrenamiento atribucional del alumnado para favorecer la sustitución de sus pensamientos de bajo control por otros de elevado control (Hall, Hladkyj, Perry y Ruthig, 2004). 
Por otra parte, asumiendo la importancia del valor de la tarea como principal antecedente en el surgimiento de emociones académicas concomitantes, nos parece primordial insistir a los docentes universitarios en que acentúen los esfuerzos por explicitar a los estudiantes el valor de las actividades que se proponen como parte de sus procesos de formación. Al respecto, sabemos que existen antecedentes de programas que han intentado potenciar entre los estudiantes la valoración de las tareas académicas desde el aula con resultados promisorios (Brophy, 2008).

Finamente, aunque el trabajo resulta interesante por sus potenciales aportes teóricos y prácticos, conviene dejar constancia de ciertas limitaciones metodológicas que pueden representar puntos de partida para investigaciones futuras. En primer lugar, considerar que si las emociones son específicas de cada asignatura (Pekrun et al., 2005), sería de gran utilidad estudiar su dinámica en el contexto de diferentes materias, en diversas carreras y distintos trayectos en el cursado de las mismas. En segundo lugar, pensamos que en sucesivas investigaciones podrían acentuarse los esfuerzos por entrevistar a todos los participantes y no solo a una muestra, esto ayudaría a recabar datos más completos acerca de un objeto de estudio tan complejo como las emociones académicas. Finalmente, sería posible obtener evidencias adicionales a estos resultados utilizando diseños longitudinales que expliquen la evolución de las emociones a lo largo de varios cuatrimestres o cursos y no solo de uno.

\section{REFERENCIAS}

Alexander, P. (2006). Psychology in Learning and Instruction. Ohio, OH: Pearson Merril Prentice Hall. Alonso Tapia, J. (2000). Motivar para el aprendizaje. Teorías y estrategias. Barcelona, España: Edebé.

Ames, C. (1992). Classrooms: goals, structures, and student motivation. Journal of Education Psychology, 84(3), 261-271.

Barab, S., y Plucker, J. (2002). Smart People or Smart Contexts? Cognition, Ability, and Talent Development in an Age Situated Approaches to Knowing and Learning. Educational Psychologist, 37(3), 165-182.

Barone, D. M. (2004). Case-Study Research. En N. Duke y M. H. Mallette (Eds.), Literacy research methodologies (pp. 7-45). Nueva York, NY: Guilford Press.

Brophy, J. (2008). Developing students' appreciation for what is taught in school. Educational Psychologist, 43, $132-141$.

CognitiveBehavioural. (12 de agosto de 2012). Inteligencia Emocional y felicidad. Entrevista a Reuven BarOn [Archivo de video]. Recuperado de http://www.youtube.com/watch?v=h5jGibf4wyE Cole, M. (1999). Psicología cultural. Madrid, España: Morata.

Csikszentmihalyi, M. (1998). Creatividad. El fluir y la psicología del descubrimiento y la invención. Barcelona, España: Paidós. 
Glaser, B., y Strauss, A. (1967). The discovery of Grounded Theory. New York, NY: Widenfeld \& Nocolson. González Fernández, A. (2005). Motivación académica. Teoría, aplicación y evaluación. Madrid, España: Pirámide.

González Fernández, A., Rinaudo, M. C., y Donolo, D. (2010). Motivación académica y ajuste emocional. Estudio en universitarios argentinos y españoles. En P. V. Paoloni, M. C. Rinaudo, D. Donolo, A. González Fernández, y N. Rosselli (Eds.), Estudios sobre motivación: enfoques, resultados, lineamientos para acciones futuras (pp. 383-403). Río Cuarto, Argentina: Universidad Nacional de Río Cuarto.

Hall, N., Hladkyj, S., Perry, R., y Ruthig, J. (2004). The role of Attributional retraining and elaborative learning in college students' academic development. The Journal of Social Psychology, 144, 591613.

Huertas, J. A. (1997). Motivación. Querer aprender. Buenos Aires, Argentina: Aique.

Huertas, J. A., y Montero, I. (2001). La interacción en el aula. Aprender con los demás. Buenos Aires, Argentina: Aique.

Linnenbrink-Garcia, L., y Pattal, E. (2016). Motivation. En L. Corno y E. Anderman (Eds.), Handbook of Educational Psychology (pp. 91-118). New York, NY: Routledge.

Mouratidis, A., Vansteenkiste, M., Lens, W., y Vanden-Auweele, Y. (2009). Beyond positive and negative affect: Achievement goals and discrete emotions in the elementary physical education classroom. Psychology of Sport and Exercise, 10(3), 336-343.

Paoloni, P. V. (2009). Contextos favorecedores de la motivación y el aprendizaje. Una propuesta innovadora para alumnos de Ingeniería. Electronic Journal of Research in Educational Psychology, 7(3), 953-984. Recuperado de http://www.redalyc.org/articulo.oa?id=293121984003

Paoloni, P. V. (2010). Motivación para el aprendizaje. Avances conceptuales e implicancias metodológicas. En P. V. Paoloni, M. C. Rinaudo, D. Donolo, A. González Fernández, y N. Rosselli (Eds.), Estudios sobre motivación: enfoques, resultados, lineamientos para acciones futuras (pp.31-38). Río Cuarto, Argentina: Editorial de la Universidad Nacional de Río Cuarto.

Paoloni, P. V. (2014). El papel de las emociones en los aprendizajes académicos. En P. V. Paoloni, M. C. Rinaudo y A. González Fernández (Comps.), Cuestiones en Psicología Educacional. Perspectivas teóricas, metodológicas y estudios de campo (pp. 83-132). Tenerife, España: Sociedad Latinoamericana de Comunicación Social. Recuperado de http://www.cuadernosartesanos.org/2014/cde01.pdf

Paoloni, P. V., Vaja, A., y Muñoz, V. (2014). Confiabilidad y validez del Achievement Emotions Questionnaire. Un estudio con alumnado universitario argentino. Electronic Journal of Research in Educational Psychology, 12(3), 671-692. Recuperado de http://www.redalyc.org/pdf/2931/293132659006.pdf 
Paoloni, P. V., y Rinaudo, M. C. (2014). Procesos de feedback desde una perspectiva multidimensional. Un estudio orientado a promover autorregulación en estudiantes universitarios. En P. V. Paoloni, M. C. Rinaudo, y A. González Fernández (Comps.), Cuestiones en Psicología Educacional. Perspectivas teóricas, metodológicas y estudios de campo (pp. 287-323). Tenerife, España: Sociedad Latinoamericana de Comunicación Social (SLCS). Recuperado de http://www.cuadernosartesanos.org/2014/cde01.pdf

Paoloni, P. V., y Rinaudo, M. C. (2015). Dinámica emocional en contextos de evaluación. Un estudio con alumnos de ingeniería. Revista Novedades Educativas, 288, 30-35.

Pekrun, R., Frenzel, A., Goetz, T., y Perry, R. (2007). The Control-Value Theory of Achievement Emotions: An Integrative Approach to Emotions in Education. En P. Schutz y R. Pekrun (Eds.), Emotion in Education (pp. 13-36). San Diego, CA: Academic Press.

Pekrun, R., Goetz, T., y Perry, R. (2005). Achievement Emotions Questionnaire (AEQ)-user's Manual. University of Munich, Alemania: Department of Psychology.

Perry, N., Turner, J., y Meyer, D. (2006). Classrooms as contexts for motivating learning. En P. Alexander y P. Winne (Eds.), Handbook of Educational Psychology (pp: 327-348). Londres, Inglaterra: Lawrence Erlbaum Associates.

Pintrich, P., y Schunk, D. (1996). Motivation in Education: theory, research and applications. New Jersey, NJ: Prentice Hall.

Preocupa la falta de motivación de los estudiantes secundarios. (21 de abril de 2002). Clarín. Recuperado de https://www.clarin.com/sociedad/preocupa-falta-motivacion-estudiantessecundarios_0_BkOT4SgRtl.html

Rinaudo, M. C. (1999). Comprensión del texto escrito. Estudios acerca de la naturaleza del proceso y las posibilidades de la instrucción. Río Cuarto, Argentina: Fundación Universidad Nacional de Río Cuarto.

Rinaudo, M. C., y Donolo, D. (2010). Estudios de diseño. Una alternativa promisoria en la investigación educativa. RED Revista de Educación a Distancia, 22, 2-29. Recuperado de http://www.um.es/ead/red/22/rinaudo_donolo.pdf

Rinaudo, M. C. (2014). Estudios sobre el contexto de aprendizaje: arenas y fronteras. En P. V. Paoloni, M. C. Rinaudo, y A. González Fernández (Comps.), Cuestiones en Psicología Educacional: aspectos teóricos, metodológicos y estudios de campo (pp. 163-206). Tenerife, España: Sociedad Latinoamericana de Comunicación Social (SLCS). Recuperado de https://issuu.com/revistalatinadecomunicacion/docs/cde01

Rinaudo, M. C., y Paoloni, P. V. (2013). Feedback en los aprendizajes. Potencialidad en los contextos virtuales. En A. Chiecher, D. Donolo, y J. L. Corica (Eds.), Entornos Virtuales y aprendizaje. Nuevas perspectivas de estudio e investigaciones (pp. 89-126). Mendoza, Argentina: Editorial Virtual Argentina. 
Rodríguez, X. (2003). La investigación y mejora de la práctica en el aula. En L. Sanjurjo y X. Rodríguez (Eds.), Volver a pensar la clase. Las formas básicas de enseñar (pp: 193-249) . Madrid, España: HomoSapiens.

Ryan, R., y Decy, E. (2000). Intrinsic and Extrinsic Motivations: Classic Definitions and New Directions. Contemporary Educational Psychology, 25, 54-67.

Sánchez, F. (4 de junio de 2013). Universidades: se reciben sólo 27 alumnos de cada 100 que ingresan. La Nación. Recuperado de https://www.lanacion.com.ar/1588252-universidades-se-reciben-solo27-alumnos-de-cada-100-que-ingresan

Schutz, P., y Pekrun, R. (2007). Emotion in Education. San Diego, CA: Academic Press.

Urdan, T., y Turner, J. (2005). Competence Motivation in the Classroom. En A. Elliot y C. Dweck (Eds.), Handbook of Competence and Motivation (pp. 297-317). New York, NY: The Guilford Press.

Vaja, A., y Paoloni, P.V. (2012). Emociones en contextos académicos. Un análisis de las emociones de logro en una asignatura del nivel superior. En M. Bonyuan, S. Peppino, M. Lerchundi, y N. Galetto (Eds.), Ciudad Moderna: problemas, conflictos, desafíos (pp. 190-194). Río Cuarto, Argentiina: Ediciones Del ICALA.

Winne, P., y Marx, R. (1989). A cognitive-processing analysis of motivation within classroom tasks. En G. Ames y R. Ames (Eds.), Research on Motivation in Education (pp. 223-257). Orlando, FL: Academic Press. 